\title{
Unintended Consequences of Hospital Payment: The Case of Swiss Diagnosis Related Groups
}

\author{
Philippe K. Widmer ${ }^{1}$, Peter Zweifel ${ }^{2 *}$ \\ ${ }^{1}$ Senior Health Economist, Polynomics AG, Baslerstr. 60, 4600 Olten, Switzerland \\ ${ }^{2}$ Department of Economics, University of Zurich, Switzerland
}

*Corresponding Author: Peter Zweifel, PhD, Emeritus, Department of Economics, University of Zurich, Switzerland. Email: peter.zweifel@uzh.ch

Received July 25, 2016; Accepted October 25, 2016; Online Published December 18, 2016

\begin{abstract}
Background: In 2012, Switzerland changed from retrospective to prospective hospital payment based on diagnosis related groups (DRGs), following the example of the United States, Australia, and Germany. As in these countries, the objective of this transition was to motivate hospitals to improve efficiency by making them bear financial risk to some extent.

Objective: This contribution seeks to find out whether SwissDRG, the Swiss version of DRG payment, indeed provides hospitals with appropriate incentives, thus creating a level playing field enabling workable competition between them.

Methods: Three conditions for creating a level playing are stated, of which the first is tested using data on some 757000 patient cases treated by 93 hospitals in the year 2012 .

Results: The evidence suggests that hospital payment as currently devised by SwissDRG fails to create a level playing field. Differences in margins over cost of treatment can be traced to a hospital's portfolio of specialties and mix of patients, both of which are largely beyond their control. The findings of this paper are subject to several limitations. The true DRG-specific cost distributions (and hence expected values) are not known; moreover, emphasis has been on variable cost, neglecting fixed (capital user) cost. Finally, hospitals with a high amount of capital user cost may well benefit from modern technology contributing to their efficiency in terms of variable cost.

Conclusion: The finding that current hospital financing by SwissDRG fails to create a level playing field is likely to be robust, calling for an expeditious adjustment be-cause hospitals are exposed to financial risk to a greatly differing degree. It may be appropriate for them to purchase insurance against their financial risk, which is largely driven by influences beyond their control.
\end{abstract}

Keywords: Hospital financing, DRG payment, Cost efficiency, Financial risk, Incentive problems

\section{Background}

1.1. Changes in Swiss Hospital Payment

As in other industrial countries, continuously increasing healthcare expenditure has spawned proposals for reform of the Swiss healthcare sector designed to dampen this surge. The transition from retrospective to prospective hospital payment constitutes one such reform, instigated in 2012. Following the example of the United States, Australia, and Germany, all patient cases admitted for acute somatic care are paid using a fixed amount (based on so-called diagnosis related groups, DRGs). Since payment does not necessarily cover actual cost of treatment anymore, hospitals are exposed to financial risk. This should motivate them to strive for cost efficiency, with the ultimate aim of limiting the increase in hospital expenditure without jeopardizing quality of treatment.

While there is some empirical evidence suggesting that cost efficiency has indeed improved, ${ }^{1}$ the question remains whether the new system can contribute to slower growth of Swiss hospital expenditure in the long run. The reason is that the current fee schedule imposed by SwissDRG fails to adequately compensate hospitals for financial risks that are beyond their control. ${ }^{2}$ DRG fees are designed to confront all efficient hospitals with the same amount of financial risk regardless of their portfolio of specialties and patient mix, which are largely determined by cantonal authorities (a canton is a member state in Switzerland). Because cantons finance roughly one-half of hospital's operational expenditure and capital investment, they wield considerable regulatory authority. If DRG payment is not commensurate with financial risk, hospitals are predicted to pursue other goals than improving cost efficiency, e.g. by selecting lucrative services and patient cases. ${ }^{3,4}$ While these strategies result immediately in higher margins over cost of treatment, they do not reduce overall hospital expenditure since they merely amount to a dumping of high-cost patients on other hospitals. Hospitals at the high end of the referral chain are likely to suffer most

Copyright (C) 2016 The Hospital Practices and Research. This is an open-access article distributed under the terms of the Creative Commons Attribution License (http://creativecommons.org/licenses/by/4.0), which permits unrestricted use, distribution, and reproduction in any medium, provided the original work is properly cited. 
because cantonal authorities typically prevent them from optimizing their portfolio of specialties and their patient mix in this way. Even though they may be as cost-efficient as their competitors, they have to cope with a higher financial risk, putting them at a competitive disadvantage.

\subsection{The Swiss Hospital Payment Formula}

Swiss lawmakers commissioned SwissDRG to develop an entirely prospective payment system based on no more than 1200 diagnostic groups that are homogenous in terms of treatment cost (in 2012, there were 967 in active use). Each case $i$ admitted for acute somatic treatment is assigned to one DRG $j$, to be paid according to a predetermined amount $F P$ that is independent of actual treatment cost, $F P_{j}=k_{j}^{*} B P$,

where $k_{j}$ symbolizes a cost weight and $B P$, a base price that is independent of the DRG. The cost weight $k_{j}$ reflects DRG-related differences in average treatment cost. These averages are calculated by SwissDRG using data provided by participating hospitals (100 in 2012). They distinguish between patients with length of stay (LOS) within predetermined bounds and those below and above these bounds. All patient cases with DRG $j$ falling within these bounds enter the calculation of $k_{j}$ in the same way; there is no differentiation according to hospital type or other hospital characteristics (such as e.g. catchment area). For patients with LOS below the lower bound, there is a fixed deduction per case, for those above the upper bound, a fixed surcharge per case. For simplicity, this deduction (surcharge, respectively) is neglected in the formula above. However, the system is designed to cover average treatment cost per DRG (for more detail, see Reference 5).

As to the base price $B P$, it shifts payment up or down across all DRGs, thus determining the share of actual percase cost that is remunerated in a given DRG category. It is the steering variable to implement incentives for cost efficiency.

\section{Objective}

The objective of this contribution is to check whether the current structure of DRG payment in Switzerland creates a level playing field by exposing efficient hospitals to comparable financial risks beyond their control (Additional detail and evidence can be found in Spika and Zweifel ${ }^{6}$ and Widmer ${ }^{7}$ ). Three conditions are stated that must be met for a hospital financing to create a level playing field in the presence of a nationally uniform DRG schedule, as is the case in Switzerland. The first condition states that DRG payment needs to cover the expected value of treatment cost per DRG category. This is the one to be tested below.

\section{Methods}

3.1. Conditions for Creating a Level Playing Field in the Presence of a Uniform Nationwide DRG Schedule Currently, the base price $B P$ is uniform across all Swiss hospitals in the SwissDRG tariff structure; it is equal to the average treatment cost calculated over patients countrywide as of 2012. In this way, SwissDRG seeks to create the conditions for fair competition between hospitals. Adjustments in $B P$ over time or between hospitals are subject to negotiations between hospitals and insurers who finance roughly one-half of hospital expenditure and investment. The remainder is covered by the canton of residence, which is not involved in the negotiation of the $B P$. The point of departure is a cost benchmark which is derived by dividing a hospital's total treatment expenditure by the sum of its cost weights. This benchmark value equals the base price $B P$ that the given hospital needs for recovering its actual cost of operation. If this cost benchmark exceeds the nationwide average (or a certain percentile of the nationwide distribution in some cantons), the hospital is deemed cost-inefficient; if it is at or below the average (or the relevant percentile, respectively), it is deemed efficient.

Hospitals above the cost benchmark are under pressure to reach it by reducing their expenditure. The burden of proof is on them if they want to justify the excess. However, in the long run the aim of SwissDRG is to achieve a fully uniform nationwide base price, causing hospitals to have an identical incentive for cost efficiency.

\subsection{Incentives for Pursuing Other Strategies Than Cost Efficiency}

Since only expected cost of treatment in a given DRG category is to be covered by DRG payment, Swiss hospitals have incentives to pursue other strategies than cost efficiency - even regardless of the outcome of negotiations concerning the base price $B P$. There are two reasons for this.

(1) Cost risk: Cost of treatment is a random variable that takes on values which can be far above expected value. Indeed, the cost distribution in many DRGs has a long tail (i.e. substantial positive skewness). Focusing on expected value and variance for simplicity, actual treatment cost per case $F K_{i j}$ follows some distribution law $p(\cdot)$,

$$
F K_{i j} \sim p\left(\mu_{j} ; \sigma_{j}^{2}\right)
$$

with expected value $E\left(F K_{i j}\right)=\mu_{j}$ and variance $\operatorname{Var}\left(F K_{i j}\right)=$ $\sigma^{2}$

In this formula, variance $\sigma_{j}^{2}$ approximates the cost risk in DRG $j$ (of course, hospital management is concerned with the positive deviations from expected value $\mu_{j}$, while variance treats positive and negative deviations symmetrically).

(2) Demand risk: The number of patients treated in a given DRG also constitutes a random variable. Yet the cost benchmark (and hence DRG payment) depends on the number of patient cases budgeted at the beginning of the year (note the role of the sum of cost weights in Section 2.2). Evidently, hospitals cannot predict with precision whether patient $i$ will need hospital services pertaining to DRG category $j$. This is a binary random variable $X_{i j}$ taking on the value of 1 (yes, the patient needs the service of category $j$ ) with probability $p j$ and 0 (no, this service is not needed) with probability (1$p j$ ). This makes the number of patient cases $n j$ in DRG category $j$ a random variable too, defined by 
$n_{j}:=\sum_{i}^{N} X_{i j}$

where $N$ denotes the population of the hospital's catchment area that potentially could be treated by the hospital. At the national level, Switzerland, $N$ equals the Swiss resident population. Assuming the $X_{i j}$ to be independent across patients, $n j$ follows a binomial distribution as well,

$n_{j} \sim B\left(p_{j}, N\right)$,

with expected value $E\left(n_{j}\right)=N p_{j}$ and variance $\operatorname{Var}\left(n_{j}\right)=N p_{j}$ $\left(1-p_{j}\right)$.

This variance can be said to approximate the hospital's demand risk.

The unintended consequences of current SwissDRG payment can be seen from analyzing the hospital's margin in excess of cost, summed over all DRG categories denoted by $I$, and given by

$I=\sum_{j=1}^{J} \sum_{i=1}^{N} X_{i j}\left[F P_{j}-F K_{i j}\right]$

Evidently, this is a random variable as well. If the number of patients treated exceeds the budgeted value while DRG payment exceeds actual cost of treatment, $I>$ 0 , indicating a positive margin. Conversely, if the number of patients treated falls short of the budgeted value while DRG payment fails to cover actual cost of treatment, $I<0$, indicating a deficit.

The cost and demand risks create a financial risk that hospitals need to optimize in order to secure their longterm economic survival. To simplify the analysis, the individual hospital is defined by its share of the national hospital market, which is represented below as though it was one single (large) hospital. In this way, the Law of large numbers can be invoked, which states that when $N$ becomes large, the binomial distribution converges to the normal one. ${ }^{2}$ At the aggregate level, one therefore has, with $N$ symbolizing the normal distribution (in this formula only),

$I \sim N(E(I), \operatorname{Var}(I)$,

with expected value given by

$$
\begin{aligned}
& E(I)=\sum_{j=1}^{J} \sum_{i=1}^{N} E\left[X_{i j}\right] E\left[F P_{j}-F K_{i j}\right] \\
& =\sum_{j=1}^{J} N p_{j}\left[F p_{j}-\mu_{j}\right]
\end{aligned}
$$

Assuming independence between $X_{i j}$ and $F K_{i j}$, the variance is given by (see http://www.odelama.com/dataanalysis/Commonly-Used-Math-Formulas/),

$$
\begin{aligned}
& \operatorname{Var}(I)=\sum_{j=1}^{J} \sum_{i=1}^{N} \operatorname{Var}\left[X_{i j}\left(F P_{j}-F K_{i j}\right)\right] \\
& +E\left[F P_{j}-F K_{i j}\right]^{2} \operatorname{Var}\left(X_{i j}\right)+E\left[X_{i j}\right]^{2} \operatorname{Var}=
\end{aligned}
$$

$\sum_{j=1}^{J} \sum_{i=1}^{N} \operatorname{Var}\left[X_{i j}\right] \operatorname{Var}\left[F P_{j}-F K_{i j}\right]\left(F P_{j}-F K_{i j}\right)$

$=\sum_{j=1}^{J} N p_{j}(1-p) \sigma_{j}^{2}+\left(F P_{j}-\mu_{j}\right)^{2} N p_{j}\left(1-p_{j}\right)+\left(N_{j} p_{j}\right)^{2} \sigma_{j}^{2}$

Now consider a particular hospital $t$. There are several ways for its management to increase (its share of) $E(I)$ and/ or decreasing (its share of) $\operatorname{Var}(I)$ :

- It can seek to decrease $\mu_{j t}$, i.e. its expected cost of treatment of patients falling in DRG category $j$. While this amounts to an improvement in its cost efficiency, it increases the hospital's cost risk, as evidenced from the second term of $\operatorname{Var}(I)$ as long as the DRG fee $F P_{j}$ remains the same.

- It can seek to reduce $\sigma_{j p}$ i.e. the uncertainty surrounding its cost of treating patients falling in DRG category $j$. One way to achieve this is to introduce treatment guidance for physicians and nursing personnel, which even may result in quality assurance. However, another way is to dump 'risky' patients on other hospitals, which has the potential of lowering cost efficiency at the system level.

- It can manipulate $N_{t}$, i.e. its share of total $N$ in a way that a high value of $N_{i,}$ its number of patients treated in DRG $j$, interacts with a low value of $\mu_{j t}$, thus boosting its share of $E(I)$. At the same time, this also serves to reduce the hospital's share in $\operatorname{Var}(I)$ very effectively because $N$ enters the last term of the formula in squared form. This can be viewed as an attempt to specialize in the treatment of conditions where the hospital has a cost advantage, a potentially beneficial effect. Note that in Switzerland, even small hospitals seek to offer a comprehensive range of specialties in an attempt to attract investment funds from their canton of residence. The downside of specialization of course is longer travel times for patients with a rare health condition.

- In addition, management can manipulate $N_{j t}$ in a way that large values interact with a low value of $\sigma_{j t}$. While not affecting the hospital's share in $E(I)$, this strategy again is very effective in reducing the hospital's share in $\operatorname{Var}(I)$ because $N_{j t}$ enters the last term of the formula in squared form. This amounts to an attempt to specialize in the treatment of less 'risky' patients or in favoring DRG categories where the hospital has implemented treatment guidance.

- Finally, the hospital can manipulate $p_{j}$, i.e. the probability with which a patient falls in DRG category $j$. If that category should be characterized by both a low value of $\mu_{j t}$ and $\sigma_{j t}$, this strategy serves to boost the (hospital's share of) $E(I)$ while decreasing (its share of) $\operatorname{Var}(I)$. Its assessment is ambiguous: On the one hand, this may amount to building up reserve capacities to meet uncertainty in local demand; on the other hand, it may reflect biased coding of treatment cases $^{6}$ or demand inducement in that patients are 'nugded' into treatment alternatives with favorable DRG payment, 
both of which are not compatible with cost efficiency.

A beneficial side effect not related to cost efficiency is that strategies that reduce the hospital's share in $\operatorname{Var}(I)$ facilitates its negotiations with the insurer. Hospital management knows with high certainty whether or not a given base price $B P_{j}$ will be sufficient to cover treatment cost in the pertinent DRG category.

Finally, the analysis performed here focuses on variable cost because SwissDRG is designed to cover the variable cost of treatment on expectation. Fixed cost is mainly capital user cost; however, cantons have been allocating capital for investment in hospitals without charging them interest and depreciation. To the extent that cantonal administrations may modify their behavior in future, capital user cost would have to be incorporated in $\mu_{\text {i }}$ without however affecting the formula. Of course, the set of strategies available to hospitals would have to be extended to also comprise investment designed to create treatment capacities in 'promising' DRG categories.

\subsection{Three Conditions for Creating a Level Playing Field} Evidently, the optimization strategies cited in the preceding section may not be conducive to an improvement of cost efficiency and a slowed surge in hospital expenditure. Given that SwissDRG pays a uniform fee for a given service, these objectives can be attained only if hospitals with efficient operations are exposed to the same amount of financial risk regardless of their portfolio of specialties and case-mix. This implies that the fee structure must fully reflect cost heterogeneity to the extent that heterogeneity does not depend on differences in hospitals' effort at cost efficiency. In terms of the formulae presented above, this amounts to the following three conditions.

(1) Neglecting hedging possibilities across DRG categories (which are limited especially for specialized hospitals), the first condition demands that in every DRG $j$, prospective payment must equal the expected nationwide value of treatment cost,

$F P_{j}=\mu_{j}$

In fact, a considerable number of DRGs contain too few treatment cases for deriving a robust estimate of $\mu_{j}$, resulting in a violation of this condition. More-over, the analysis performed here argues for risk-adjusted cost weights, as calculated by Lüthi and Widmer. ${ }^{2}$ Without risk adjustment, violations of this condition become frequent and substantial, creating an incentive for hospitals to favor 'promising' DRG categories rather than striving for cost efficiency.

(2) Each DRG category must give rise to a comparable amount of financial risk, which in addition must be supportable to a hospital of average size, with average size defined by $\bar{n}=1 / T \sum_{j=1}^{J} n_{j}$, the aggregate of all patients treated in all DRG categories divided by the number of hospitals $T$. Since the average Swiss hospital treats rather few patients (indicated by $\bar{n}=8360$ in 2012), the financial risk relative to expected cost and per case treated must also be small in each DRG category, $\frac{\sigma_{j}^{2}}{\bar{n}} \cong 0$

Note that this condition refers to the overall number of cases treated rather than those in DRG $j$ in order to account for the possibility of hedging risk across DRG categories (this is far simpler than introducing covariances between deviations from $\mu_{j}$ ). This second condition needs to be imposed because currently SwissDRG does not remunerate the user cost of capital needed to provide hospitals with a financial reserve. Although capital user cost is not currently charged by several cantons, it does enter informally in negotiations about investment in upgrading facilities or expansion of capacities. Anticipating this, hospital management may well pursue the strategies of patient selection and demand inducement described in the preceding section rather than making effort at cost efficiency.

(3) The averaging procedure performed by SwissDRG creates a level playing field only if the probability distribution of actual treatment costs in a given DRG $j$ is identical across hospitals and patient cases (note the absence of subscript $i$ for $p$ in the formula below),

$$
F K_{i j} \sim p\left(\mu_{j}, \sigma_{j}^{2}\right)
$$

Note also the absence of any influence emanating from a DRG category other than $j$, indicating independence. In particular, this means that cases cannot be split between categories in an attempt to maximize the volume of services charged. Failing this condition, the cost weights are biased; moreover, hospital management has again leeway to improve its financial status by engaging in patient selection and demand inducement rather than striving for cost efficiency.

Failure of only one of these three conditions to be satisfied creates an incentive for Swiss hospitals to secure their economic survival by other means than improving cost efficiency. Indeed, they may often find it easier to focus on 'promising' DRG categories, selecting lucrative patients, and simply increasing the number of cases treated (thus benefiting from the Law of large numbers and hence decreased variance). However, this means that cost-efficient hospitals are exposed to differing amounts of financial risk. Some may even incur a deficit in spite of being cost-efficient, while hospitals neglecting cost efficiency may achieve a high margin over treatment cost. In sum, current SwissDRG hospital payment does not seem to create a level playing field.

\subsection{Data Base}

The data were made available by the Federal Statistical Office (BFS); they correspond to those used by Widmer. ${ }^{7}$ Their structure follows catalogue 3.0 of SwissDRG categories as of 2012; these data were used for the calculation of cost weights applicable in 2014. However, in the analysis below several hospitals and certain observations had to be excluded, resulting in small deviations from the official data base. 
As shown in Table 1, there were 100 participating hospitals, all of them engaged in acute somatic treatment. Their data were checked in terms of quality and completeness, in the same way as in Widmer. ${ }^{7}$ This resulted in the exclusion of seven hospitals and 38261 (of 794908) observations. Therefore, the sample consists of five university hospitals (most of the 26 cantons do not have a university), 20 so-called central hospitals (typically hospitals serving an entire canton that lacks a university hospital), 50 regional hospitals, and 18 specialized clinics (e.g. for the treatment of workplace accidents). In all, the retained observations correspond to $80 \%$ of hospital services provided in Switzerland.

\subsection{Testing Methodology}

Recall that the condition to be tested calls for the equality of the DRG fee $F P_{j}$ with the countrywide expected value of treatment cost $\mu_{j}$. Violation of this condition results in positive and negative margins $\bar{I}_{j}$, defined by

$\bar{I}_{j}=\frac{1}{n_{j}^{*}} \sum_{i=1}^{n_{j}^{*}}\left(F P_{j}-F K_{i j}\right)$.

Here, $n^{*}$ symbolizes the actual number of cases treated in DRG $j$ and FKij, the actual cost of treatment incurred by hospital $t$. Total remuneration for treatment in category $j$ (the summation of the $F P_{j}$ terms) can be calculated by multiplying the uniform base fee (which equals average normed treatment cost of all participating Swiss hospitals) by the average cost weight. This calculation corresponds to that performed by SwissDRG for the year 2014.

Formally, the hypothesis to be tested reads

$H_{0}: E\left[\bar{I}_{j}\right]=0$

against

$H_{1}: E\left[\bar{I}_{j}\right] \neq 0$

Hypothesis $H_{0}$ can be tested using a $t$ test, except in the case of 50 DRG categories where the number of cases treated falls short of 30 .

\section{Results}

As becomes evident from Figure 1, the hypothesis $H_{0}$ must be rejected for a majority of DRG categories. Both positive and negative margins attain values of up to $\mathrm{CHF}$
15000 - a non-negligible quantity with the average DRG fee amounting to CHF 9800 per case treated. Note that the $\mathrm{x}$-axis of Figure 1 depicts the importance of the DRG, measured by the number of cases treated (21 DRGs with more than 5000 cases not shown). Clearly, 'small' DRGs are associated with a particularly high financial risk, indicating violation of condition No.1. In addition, with the average Swiss hospital active in no more than 500 categories, condition No. 2 (variance close to zero) is likely not to be satisfied either (a stronger statement would require a statistical test).

There are two main causes for the failure of SwissDRG to satisfy condition No. 1 for creating a level playing field (for more detail, see Widmer ${ }^{7}$ ).

\subsection{Too Few Patient Cases in the DRG Categories}

According to the Law of large numbers, robust cost weights can be calculated only if the number of cases treated is large. Otherwise, DRG fees can deviate strongly from the expected value of cost; in addition, they may exhibit substantial variation over time. These effects not only boost a hospital's financial risk in a given year but also reduce predictability of its future revenue.

Figure 1 indicates that these problems emerge in DRG categories with fewer than 1000 patient cases, affecting about 800 of the 957 DRGs. The relationship between variance in cost weights and number of cases treated can be further investigated by relating the two in the case of two specific DRGs, N21Z (low cost variability) and E77C (high cost variability). Figure 2 shows the results of a Monte Carlo-type simulation. First, actual cost weights

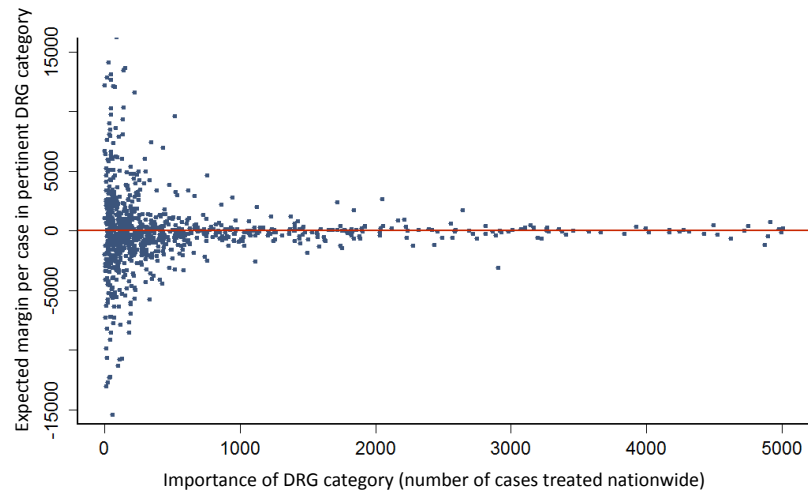

Figure 1. Margins Per Case Treated as a Function of the Importance of the DRG Category (in CHF, 1 CHF = US\$1.04 as of 2012).

Source: Swiss Federal Statistical Office (BFS) and authors' calculations.

Table 1. Observations According to Type of Hospital, Switzerland (2012)

\begin{tabular}{|c|c|c|c|c|}
\hline \multirow{2}{*}{ Hospital Type } & \multicolumn{2}{|c|}{ Number of Units } & \multicolumn{2}{|c|}{ Number of Patients Treated } \\
\hline & Prior to Cleaning & After Cleaning & Prior to Cleaning & After Cleanimg \\
\hline University hospitals (K111) & 5 & 5 & 154402 & 146732 \\
\hline Central hospitals (K112) & 20 & 20 & 366402 & 350654 \\
\hline Regional hospitals (K12) & 51 & 50 & 238876 & 228952 \\
\hline Specialized clinics (K23) & 24 & 18 & 35228 & 30309 \\
\hline Total & 100 & 93 & 794908 & 756647 \\
\hline
\end{tabular}

Note: In none of the hospitals and clinics, more than 5 percent of cases treated were excluded to arrive at the sample used, permitting robust statistical inference at the level of individual hospitals.

Source: Swiss Federal Statistical Office (BFS) and authors' calculations. 
pertaining to only 10 patient cases were drawn from the distribution of the respective DRG. Next, the number of draws was increased to 50 , then to 100 , then to 500 and finally, to 1000 . For simplicity, the range (maximum minus minimum) rather than the variance is depicted.

In the left-hand side panel, the estimated range of cost weights is 0.8 (relative to an average cost weight of 1.00) if the number of draws is as low as 10 , reflecting a hospital that has but a few cases in this category. With 50 draws, the range decreases to 0.4 , and with 1000 draws (reflecting a large or very specialized hospital), it is as low as 0.05 . By way of contrast, the panel on the right-hand side exhibits E77C, a DRG with high cost variability. Here, the range estimated on the basis of 10 draws from the distribution of cost weights amounts to no less than 3.1, again to be compared with an average cost weight of 1.00 . However, with 1000 draws, the range falls to 0.15 . Now consider a hospital fully specialized in the treatment of patients falling in DRG categories of the E77C type, with few patients in each. It may easily exposed to an unpredictable increase or decrease in revenues by a factor of three from one year to the next.

\subsection{Deduction (Surcharge) for Length of Stay Below (Above) Thresholds}

Contrary to the Law of large numbers, discrepancies between DRG payment and expected value of treatment cost persist even in important DRG categories. This is due to the way Swiss DRG proceeds when calculating the cost weights. As detailed in Widmer, ${ }^{7}$ SwissDRG bases its calculation of the deduction (surcharge, respectively) for patient cases that are out of bounds exclusively on expenditures that vary with LOS such as hotel-type services and board (see Section 2.1 again). This implicitly supposes that the cost of medical treatment of these patients equals that of those of within-bounds LOS. Failure of this condition to hold true may generate systematic positive or negative margins. In contradistinction with the case of 'small' DRG categories, these are predictable deviations that can be exploited strategically by hospital management.

The importance of this consideration is illustrated

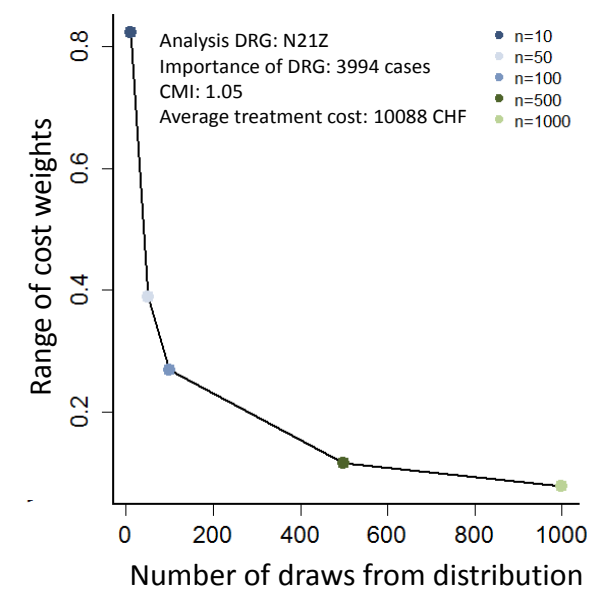

by Figure 3, which exhibits margins (in percent of the average DRG fee) for 'high outliers' (i.e. patients with LOS above the upper bound), 'inliers', and 'low outliers' (i.e. patients with LOS below the lower bound defined for the pertinent DRG category). In order to limit the influence of extreme outliers (the $5 \%$ with maximum positive and negative deviations are not shown), the median rather than the mean is used. Not even for the inliers is the median close to zero. This is approximately true also for the low outliers, who in addition give rise to limited financial risk because $90 \%$ of the margins they give rise to lie between $-50 \%$ and $+50 \%$ of the pertinent DRG fee. The picture is entirely different for the high outliers, whose median margin is $-40 \%$, with $90 \%$ of all margins bounded by close to $-100 \%$ and $+50 \%$, respectively. Evidently, they differ from the inliers not only in terms of their LOS-related cost but also in terms of their cost of medical treatment. As long as these costs are neglected in the calculation of cost weights, DRG payment will continue to deviate from the expected cost of treatment. Indeed, the higher the share of low outliers in a DRG, the higher the probability of DRG payment exceeding expected cost (and vice versa), creating an incentive for hospital management to attract cases with short LOS even though this may be medically counterindicated.

\section{Discussion}

5.1. Assessment of the Importance of Financial Risk Up to this point, no distinction between hospital types has been made. Such a distinction calls for an adjustment of patient cases in terms of case-mix because university hospitals in particular specialize in the treatment of patients with severe health conditions. Therefore, the average margin $\bar{I}_{t}$ of hospital $t$ relative to its DRG revenue from patients with cost weight 1.00 is calculated, using casemix-adjusted patient cases $n_{j t}^{* *}=n_{j t}^{*} k_{j}$ in the numerator,

$$
\bar{I}_{t}=\frac{\sum_{j=1}^{J} n_{j t}^{*} \bar{I}_{j}}{\sum_{j=1}^{J} n_{j t}^{* *}}
$$

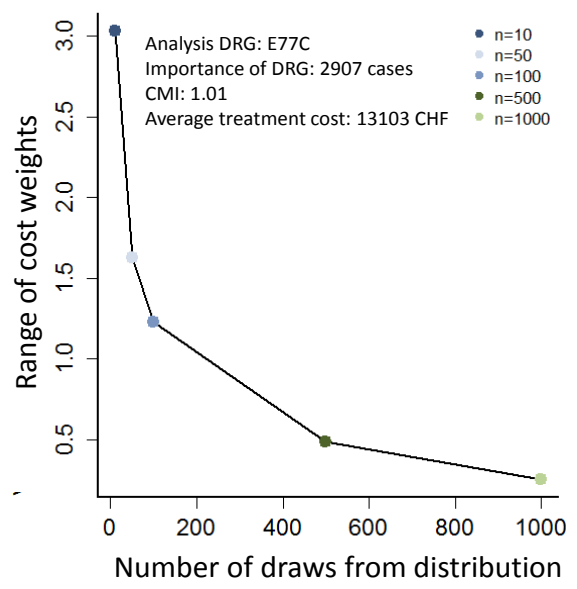

Figure 2. Simulation Designed to Estimate the Range of Cost Weights in DRGs N21Z and E77C. Source: Swiss Federal Statistical Office (BFS) and authors' calculations. 


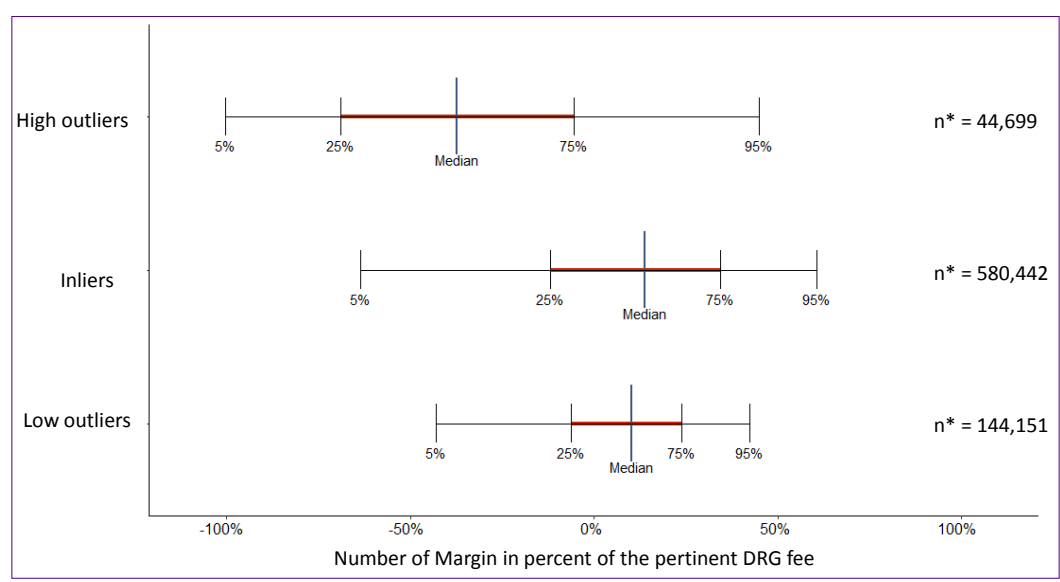

Figure 3. Margins (in \% of DRG Fee) Associated With High Outliers, Inliers, and Low Outliers. Source: Swiss Federal Statistical Office (BFS) and authors' calculations.

In Figure 4, this quantity is depicted as a function of hospital size measured by the number of patients treated per year. By definition, the average Swiss hospital has an average margin equal to zero. The five university hospitals treat at least 30000 patients each; they achieve a zero or slightly positive margin after case-mix adjustment. Central hospitals operate in the domain of 10000 to 40000 patient cases. They are confronted with margins between +100 and $-200 \mathrm{CH}$ per reference case with cost weight 1.00 . Most regional hospitals, while highly heterogeneous, are relatively small, having between 500 and 10000 patients per year. Their margins range from +220 to $-400 \mathrm{CHF}$ per case. Specialized clinics benefit most from current SwissDRG payment; their margins attain up to $400 \mathrm{CHF}$ per case and are except of two cases, which make a loss of $-600 \mathrm{CHF}$ per case, never negative. In contrast, children's hospitals are predominantly on the negative side, with margins as low as $-150 \mathrm{CHF}$ per case. In view of the fact that the margin $\bar{I}_{t}$ is calculated as the ratio of (adjusted) average DRG revenue over average treatment cost, these discrepancies are surprising indeed.

As was to be expected, small hospitals with their limited portfolio of specialties are confronted with the highest amounts of financial risk. Both maximum positive and negative margins occur in specialized clinics. The larger the hospital and more diversified its portfolio of services offered, the less it benefits (or suffers, respectively) from the biases inherent in current DRG payment. Still, when benchmarked against the most profitable private clinics, university clinics and large central hospitals may exhibit a cost disadvantage of CHF 400 per case, which for 40000 cases (say) amounts to CHF $16 \mathrm{mn}$. This should not be compared to their turnover (which is in the billions of CHF) but to SwissDRG's objective of a zero margin.

\subsection{Limitations}

The findings reported here are subject to several limitations. First, the true DRG-specific cost distributions (and hence expected values) are not known. One possibility of estimating them would be to obtain data covering several years, with the proviso however that technological change in medicine does not shift them over time. Second, in keeping with the fact that the canton of residence finances part of hospital investment, emphasis has been on variable cost. Thus when it comes to demonstrate cost efficiency with regard to fixed cost (mainly user cost of

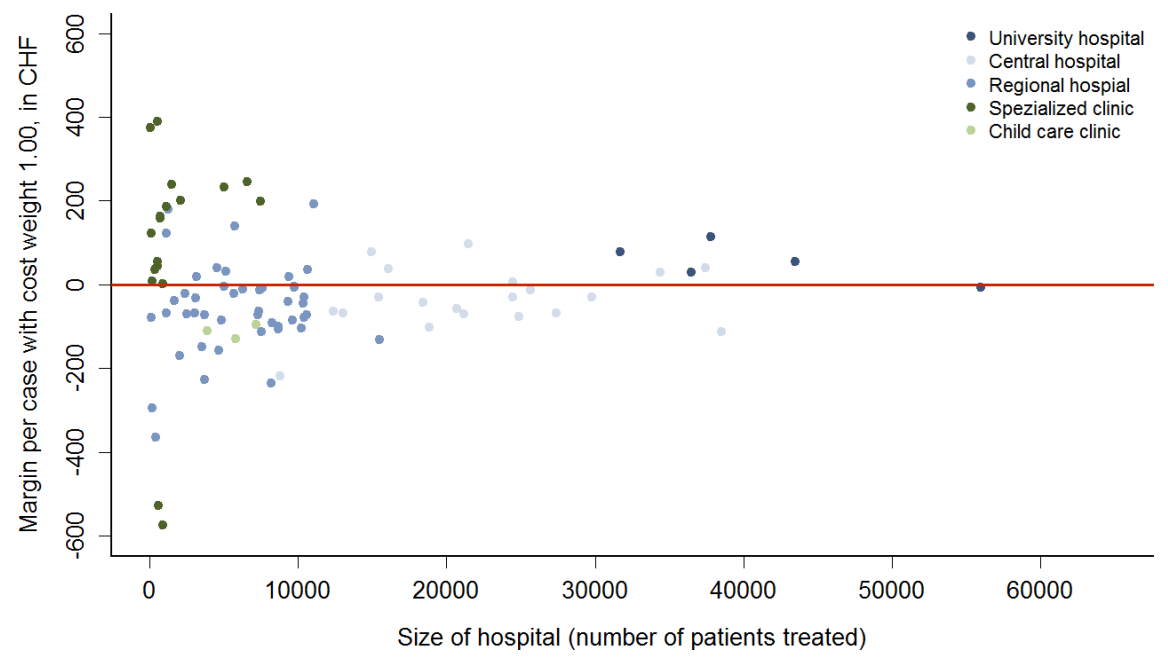

Figure 4. Expected Margins Per Case According to Type of Hospital, Case-Mix Adjusted (Switzerland, 2012) 
capital), hospital management has additional strategies at its disposal that are not discussed here. Third, hospitals with a high amount of capital user cost may well benefit from modern technology contributing to their efficiency in terms of variable cost. Hospitals whose management has been particularly successful in attracting investment funds from its canton of residence may therefore appear costefficient, a source of measurement error that is neglected in this work.

\section{Conclusion}

This contribution seeks to find out whether current hospital payment in Switzerland achieves its objective of creating a level playing field and hence the same incentive for cost efficiency for all hospitals. It formulates three conditions that must be met for SwissDRG with its uniform base fees to fully take into account heterogeneities that are beyond hospitals' control. Failing one of these conditions, hospital management has leeway to improve its financial situation by other means than improving cost efficiency. The choice of strategies includes a focus on lucrative services, patient selection, dumping loss-making cases by lowering reserve capacities, and inducing demand for treatment with high margins. Implementation of these strategies may well be easier than striving for cost efficiency, which often meets with resistance by employees.

The first of these condition states that each DRG is to be paid according to the expected cost of treatment. Its test is based on some 757000 patient cases treated in 93 hospitals who provide SwissDRG with the 2012 data to calculate DRG fees for 2014. Yet some DRGs are found to generate positive and negative margins of up to CHF 15000 (1 $\mathrm{CHF}=\mathrm{US} \$ 1.04$ as of 2012) per case, indicating that this condition is not satisfied. While margins are maximum in 'small' DRGs comprising less than 1000 patient cases (which account for no less than $80 \%$ of the total), they persist in 'large' DRGs. This failure can be traced to two causes, which could be easily remedied by SwissDRG:

(1) There has been a tendency (favored by Swiss hospitals) towards an ever finer categorization of DRGs, causing more than 300 out of 957 to contain fewer than 100 patient cases. Therefore, calculated cost weights can deviate substantially from their expected value between one year and the next, making revenue planning difficult for hospital management.

(2) Since patients with LOS below and above predetermined bounds are paid using fixed deductions and surcharges, respectively, differences in their cost of medical treatment are not taken into account by SwissDRG. 'High outliers' turn out to have higher costs than 'inliers' and 'low outliers', resulting in systematic (negative) margins for hospitals treating them.

These discrepancies have an impact on the competitiveness of Swiss hospitals, who benefit from positive or suffer from negative margins, depending on their portfolio of specialties and services offered. In particular, some specialized clinics achieve margins of up to CHF 400 per case treated without being more costefficient than their competitors. At the same time, the
Review Highlights

What Is Already Known?

Three conditions for creating a level playing field for hospitals under prospective payment are derived.

\section{What This Study Adds?}

The first, stating that prospective payment must cover the expected value of treatment cost in a DRG category, is tested using some 800000 observations covering about 100 Swiss hospitals.

This condition is found to be violated in a majority of DRG categories, causing hospitals to be exposed to major financial risk calling for insurance coverage.

hospital with the maximum negative margin of CHF -600 per case is also highly specialized; at present it is deemed highly cost-inefficient if pitted against the clinic with its CHF 400 margin. For large hospitals, the discrepancy between DRG payment and average cost of treatment can add up to millions of $\mathrm{CHF}$ revenue, although the aim of SwissDRG are margins equal to zero.

In spite of the limitations cited in Section 5.2, the conclusion that current hospital financing by SwissDRG fails to create a level playing field is likely to be robust. This finding calls for an adjustment in the mode of financing which should occur rather soon because economic survival is at stake for several Swiss hospitals (who are not necessarily cost-inefficient). As long as the base fee continues to be uniform, hospitals are exposed to financial risk to a greatly differing degree. Indeed, it may be appropriate for them to purchase insurance against their financial risk, which is largely driven by influences beyond their control (for other proposals, see Lüthi and Widmer, ${ }^{2}$ and Widmer et $\mathrm{al}^{8,9}$ ).

\section{Conflict of Interest Disclosures}

None.

\section{Ethical Approval}

Not applicable.

\section{Authors' Contributions}

All authors contributed equally to this research.

\section{References}

1. Widmer PK. Does prospective payment increase hospital (in) efficiency? Evidence from the Swiss hospital sector. Eur J Health Econ. 2015;16(4):407-419. doi:10.1007/s10198-014-0581-9

2. Lüthi HJ, Widmer PK. DRG system design: a financial risk perspective (Working paper). Zurich: Eidgenössische Technische Hochschule (ETH); 2016.

3. Ellis RP. Creaming, skimping and dumping: Provider competition on the intensive and extensive margins. J Health Econ. 1998;17(5):537-55. doi:10.1016/S0167-6296(97)00042-8

4. Ellis RP, McGuire TG. Hospital response to prospective payment: Moral hazard, selection, and practice-style effects. J Health Econ. 1996;15(3):257-277. doi:10.1016/01676296(96)00002-1

5. SwissDRG. Berechnungsmethode: Ermittlung der Kostengewichte, Ausreisser, Tarifstruktur (Method of 
calculation: cost weights, outliers, and fee structure). Bern: SwissDRG AG; 2011.

6. Spika S, Zweifel P. Internal decision-making processes and hospital behavior: hierarchical principal-agent vs. bargaining models. University of Konstanz working paper; 2016

7. Widmer PK. SwissDRG: Ein Vergütungssystem mit ungleichen finanziellen Risiken für die Spitäler (SwissDRG: a payment system with unequal financial risk for the hospitals). Swiss J Bus Res Pract. 2016;3:210-226.
8. Widmer PK, Spika S, Telser H. Leistungsorientierte Vergütung mit dem Fallpauschalensystem SwissDRG - Gleicher Preis für gleiche Leistung? (Prospective efficiency-based hospital payment by SwissDRG: Same fee for same service?) Olten: Polynomics; 2015.

9. Widmer PK, Trottmann M, Telser H. Das Fallpauschalenmodell Leistungsbezogene Basispreise unter SwissDRG (Efficiencybased prospective payment using base fees as calculated by SwissDRG). Olten: Polynomics; 2015. 